Barium iodide single-crystal scintillator detectors

N.J. Cherepy, G.Hull, T.R. Niedermayr, A. Drobshoff, S.A. Payne, U.N. Roy, Y. Cui, A.Bhattacharaya, M. Harrison, M. Guo, M. Groza, A.Burger

August 7, 2007

SPIE- Hard X-Ray and Gamma-Ray Detector Physics IX San Diego, CA, United States August 26, 2007 through August 30, 2007 
This document was prepared as an account of work sponsored by an agency of the United States Government. Neither the United States Government nor the University of California nor any of their employees, makes any warranty, express or implied, or assumes any legal liability or responsibility for the accuracy, completeness, or usefulness of any information, apparatus, product, or process disclosed, or represents that its use would not infringe privately owned rights. Reference herein to any specific commercial product, process, or service by trade name, trademark, manufacturer, or otherwise, does not necessarily constitute or imply its endorsement, recommendation, or favoring by the United States Government or the University of California. The views and opinions of authors expressed herein do not necessarily state or reflect those of the United States Government or the University of California, and shall not be used for advertising or product endorsement purposes. 


\title{
Barium iodide single-crystal scintillator detectors
}

\author{
Nerine J. Cherepy,* Giulia Hull, Thomas R. Niedermayr, \\ Alexander Drobshoff, Stephen A. Payne \\ Lawrence Livermore National Laboratory, Livermore, CA 94550, USA \\ Utpal N. Roy, Yunlong Cui, Ajanta Bhattacharaya, Melissa Harrison, \\ Mingsheng Guo, Michael Groza, Arnold Burger* \\ Center for Physics and Chemistry of Materials, Fisk University, Nashville, TN 37208, USA
}

\begin{abstract}
We find that the high-Z crystal Barium Iodide is readily growable by the Bridgman growth technique and is less prone to crack compared to Lanthanum Halides. We have grown Barium Iodide crystals: undoped, doped with $\mathrm{Ce}^{3+}$, and doped with $\mathrm{Eu}^{2+}$. Radioluminescence spectra and time-resolved decay were measured. $\mathrm{BaI}_{2}(\mathrm{Eu})$ exhibits luminescence from both $\mathrm{Eu}^{2+}$ at $420 \mathrm{~nm}$ ( 450 ns decay), and a broad band at $550 \mathrm{~nm}$ ( $\sim 3 \mu$ decay) that we assign to a trapped exciton. The $550 \mathrm{~nm}$ luminescence decreases relative to the $\mathrm{Eu}^{2+}$ luminescence when the Barium Iodide is zone refined prior to crystal growth. We also describe the performance of $\mathrm{BaI}_{2}(\mathrm{Eu})$ crystals in experimental scintillator detectors.
\end{abstract}

Keywords: Scintillators, barium iodide, $\mathrm{BaI}_{2}$, gamma ray detectors

\section{INTRODUCTION}

Detection sensitivity for weak gamma ray sources and rapid unambiguous isotope identification is principally dependent on energy resolution, and is also be enhanced by high effective atomic number of the detector material. The inorganic scintillator currently providing the highest energy resolution is $\operatorname{LaBr}_{3}(\mathrm{Ce}), \sim 2.6 \%$ at $662 \mathrm{keV}$ [1-3], but it is highly hygroscopic and its growth is still challenging. Barium Iodide is a candidate material offering higher $Z\left(\rho=5.1 \mathrm{~g} / \mathrm{cm}^{3}\right.$ and $\left.Z_{\text {eff }}=54.1\right)$ and equivalent or higher light yield than $\mathrm{LaBr}_{3}(\mathrm{Ce})$.

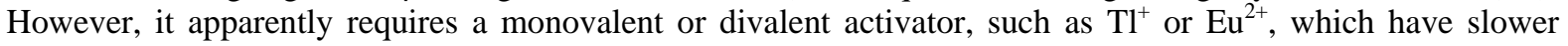
decay times compared to Ce-doped scintillators.

High light yields are found with many iodide scintillators, such as $\mathrm{CsI}(\mathrm{Tl})(65,000 \mathrm{Ph} / \mathrm{MeV})$ and $\mathrm{LuI}_{3}(\mathrm{Ce})$ (about 100,000 $\mathrm{Ph} / \mathrm{MeV}$ ) [4, 5]. Many reports of scintillation from the family of alkaline earth halides have been published, originating with the work of Hofstadter on Calcium Iodide, in the 1960's [6] which exhibits light yields in the vicinity of 100,000 Photons/MeV and has been activated with many dopants, including $\mathrm{Tl}^{+}$ and $\mathrm{Eu}^{2+}$, though it is nearly impossible to grow substantial $\mathrm{CaI}_{2}$ crystals due to its platelet growth habit. Efforts to demonstrate scintillation from Barium halides have indicated promising results, particularly for $\mathrm{BaBr}_{2}(\mathrm{Eu})$ measured at 32,000 $\mathrm{Ph} / \mathrm{MeV}$ [7]. A few reports of scintillation from unactivated $\mathrm{BaI}_{2}$ have appeared [8, 9], while the efforts of Selling, et al. to observe scintillation from $\mathrm{Eu}^{2+}$ doped $\mathrm{BaI}_{2}$ were discouraging, with only $2,000 \mathrm{Ph} / \mathrm{MeV}$ [7]. We report here the scintillation characteristics of undoped, Cerium doped, and Europium doped Barium Iodide crystals. We find that zone refining the starting material results in very different scintillation spectra. Since we find that $\mathrm{BaI}_{2}$ is a readily growable crystal with adequate mechanical properties and excellent overall light yield, we are modifying crystal growth parameters in order to produce a scintillator crystal with proportional, high light yield, and an acceptably fast decay in the blue spectral region where standard bialkali PMTs function well.

*cherepy1@llnl.gov; 925-424-3492; fax 925-423-8772; MS-L091, Lawrence Livermore National Laboratory, Livermore,CA 94550; aburger@fisk.edu; 615-329-8516; fax 615-329-8634; 1000 Seventeenth Avenue North, Dubois Hall Rm. 240, Nashville, TN 37208-3051 


\section{EQUIPMENT AND METHODS}

The single crystals of Barium Iodide were grown by the Bridgman technique at Fisk University. The melting point of $\mathrm{BaI}_{2}$ is $711^{\circ} \mathrm{C}$ and it possesses orthorhombic symmetry. Barium Iodide as-supplied powder, $99.995 \%$ pure ultra dry (Alfa Aesar) was yellowish. Crystals grown directly from the as-supplied powders retained a dark coloration. Zone refining was found to render the starting powders colorless, and resulting pure powders were used for the growth of several crystals.

Beta radioluminescence employed a ${ }^{90} \mathrm{Sr}$ source (beta endpoint energy $0.546 \mathrm{MeV}$ ). Radioluminescence spectra were collected with a Princeton Instruments/Acton Spec 10 spectrograph coupled to a thermoelectrically cooled CCD camera. A flashlamp pumped Nd:YAG laser at $10 \mathrm{~Hz}$ was operated at the $4^{\text {th }}$ harmonic of $266 \mathrm{~nm}$, producing 20 ns FWHM pulses to excite the sample. Luminescence is collected with a monochromator coupled to an R928 Hamamatsu PMT and read out by an oscilloscope.

The scintillation light produced by the samples was detected by a commercially available Hamamatsu R980 bialkali PMT. The scintillators were optically coupled to the PMT by means of mineral oil and wrapped with several layers of Teflon tape. For all measurements, the scintillator was placed in the center of the entrance window of the PMT because in this region the photocathode has better uniformity and photoelectron collection at the first dynode is optimized. The signals from the PMT anode were collected on a $500 \Omega$ load resistor, shaped with a Tennelec TC 244 spectroscopy amplifier (set with a shaping time equal to $8 \mu$ s for all the tested crystals) and then recorded with the Amptek MCA8000-A multi-channel analyzer for offline analysis.

\section{RESULTS AND DISCUSSION}

In this report, we describe the scintillation from Europium-doped Barium Iodide crystals grown from asreceived powders and from zone refined materials (Figure 1). Additionally, we describe results from undoped Barium Iodide and Cerium-doped Barium Iodide, both grown from zone refined precursor.

\subsection{Radioluminescence Spectra and Decay Times}

We actuired the beta-excited luminescence of two $\mathrm{BaI}_{2}$ crystals, both with $\mathrm{Eu}^{2+}$ doping of 0.5 mole percent, one of which was grown from zone refined (ZR) stock,; they are compared to a CsI:Tl standard crystal. The $\mathrm{Eu}^{2+}$ luminescence at $\sim 420 \mathrm{~nm}$ is enhanced in the ZR crystal, while the broad $\sim 550 \mathrm{~nm}$ band is reduced significantly (Figure 2). It is notable that the overall light yield is higher for the impurity-containing crystal; its integral light yield (including both the $420 \mathrm{~nm}$ band and the $550 \mathrm{~nm}$ band) is about $60,000 \mathrm{Ph} / \mathrm{MeV}$. In Figure 3, the betaexcited luminescence of undoped $\mathrm{BaI}_{2}, \mathrm{BaI}_{2}(\mathrm{Ce})(0.5 \mathrm{~mol} \%), \mathrm{BaI}_{2}(\mathrm{Eu})(0.5 \mathrm{~mol} \%)$ and $\mathrm{BaI}_{2}: \mathrm{Eu}(1.5 \mathrm{~mol} \%)$ are shown for comparison. The weak band at $550 \mathrm{~nm}$ may be assigned to a trapped exciton, typically such luminescence is facilitated by the presence of impurity species. This is in good agreement with the reduction in the $550 \mathrm{~nm}$ band for zone refined crystals, compared to crystals grown from unrefined materials.

The alpha-excited luminescence of the ZR Ce-doped and the Eu-doped $\mathrm{BaI}_{2}$ crystals show only luminescence consistent with $\mathrm{Ce}^{3+}$ and $\mathrm{Eu}^{2+}$ luminescence (Figure 4). Possible reasons include: (1) the de-excitation pathway for the higher $\mathrm{dE} / \mathrm{dx}$ of alphas may favor the dopant luminescence, (2) the surface layer probed by alphas may differ in doping level or otherwise chemically from the bulk, resulting in a different observed spectrum.

Time resolved luminescence reveals that the $\mathrm{Eu}^{2+}$ band decays with a submicrosecond time constant (Figure 5), while the trapped exciton band is very long-lived, and cannot be fully integrated within a reasonable shaping time $(<12 \mu \mathrm{s})$. It is interesting that the trapped exciton forms after excitation is transferred from the $\mathrm{Eu}^{2+}$, as revealed by rise-time observed for $600 \mathrm{~nm}$ detection, and that it decays very slowly, on the tens of microseconds timescale (perhaps due to the exciton experiencing a triplet to singlet spin-forbidden transition).. For the ZR crystal, the $\mathrm{Eu}^{2+}$ decay is lengthened to $770 \mathrm{~ns}$, which is still reasonable for scintillation counting. Further 
improvements are likely with higher $\mathrm{Eu}^{2+}$ doping and the use of zone refined materials, such that light yields in the $\mathrm{Eu}^{2+}$ band could reach $>60,000 \mathrm{Ph} / \mathrm{MeV}$.

\subsection{Pulse Height Spectra}

The gamma ray pulse-height spectra of the $662 \mathrm{keV}$ line of the Cs-137 radioactive source have been acquired with the Barium Iodide crystals and with a commercial CsI:Tl for comparison. The total absorption peaks have been processed with a Gaussian fit procedure to evaluate the peak position and the peak full width at half maximum, in order to estimate the scintillation light yield and the energy resolution, respectively. In Figure 6 the pulse-height spectrum of a $\mathrm{BaI}_{2}(\mathrm{Eu})(0.5 \mathrm{~mol} \%)$ crystal is reported. Table 1 summarizes results of pulse height spectra acquired for all the crystals described above. The discrepancy between the integral light yield and the achievable energy resolution is in part due to delayed luminescence components, as well as poor spectral overlap between luminescence in the $550 \mathrm{~nm}$ band and the PMT quantum efficiency.

\subsection{Conclusions}

The high light yields observed under beta excitation and the improved light yield into the $\mathrm{Eu}^{2+}$ band with higher doping and zone refined materials suggest that Barium Iodide crystals could offer high energy resolution gamma ray spectroscopy.
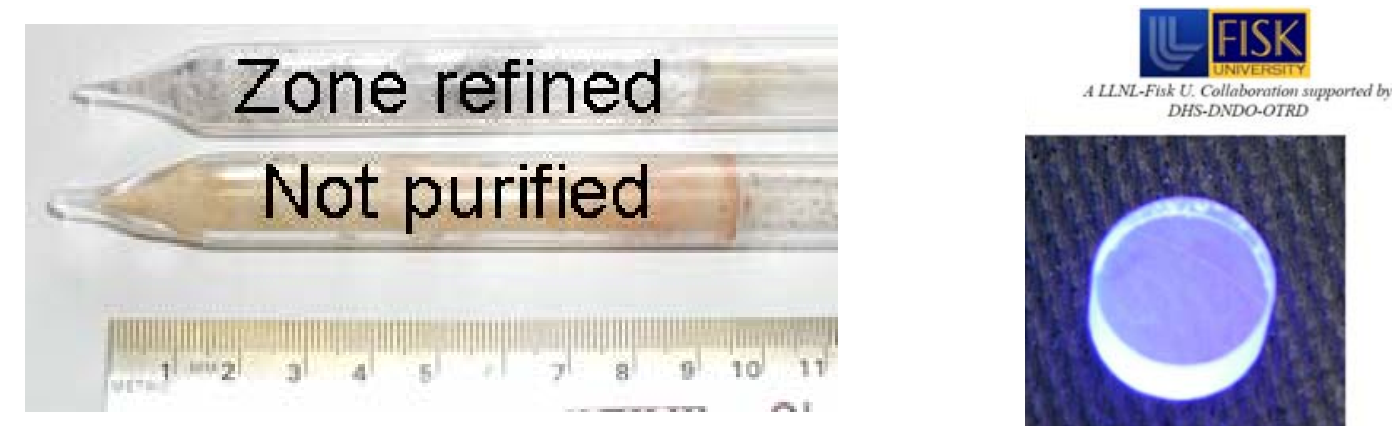

Fig. 1. (left) Photograph of two Barium Iodide boules, showing the removal of a brownish coloration upon zone refining. (right) $\mathrm{A} \mathrm{BaI}_{2}(\mathrm{Eu})$ crystal grown from zone-refined materials under room lights and under UV illumination. 


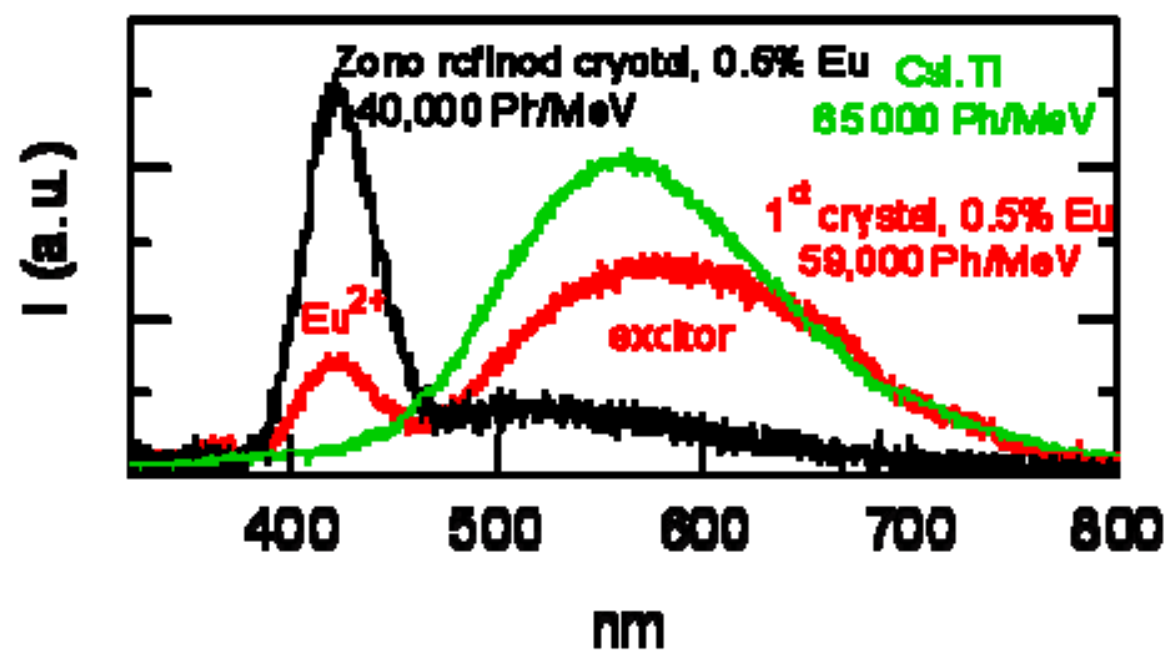

Fig. 2. Beta-excited radioluminescence spectra acquired of a two Eu-doped Barium Iodide crystals, one grown from as-received powders (black), the other from zone refined materials (red). Light yield is compared with that of Tl-doped Cesium Iodide (green), and may be compared in an absolute sense.

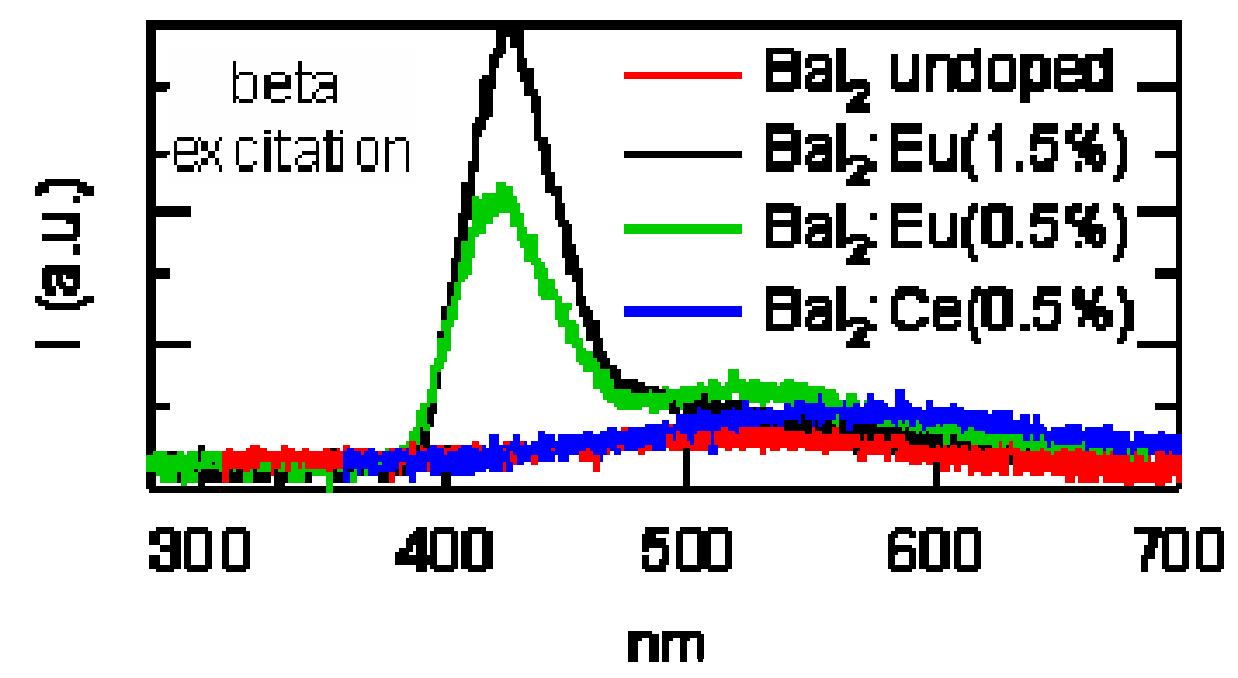

Fig. 3. Beta-excited radioluminescence spectra acquired of Barium Iodide crystals, all grown from zone refined materials. Light yields may be compared in an absolute sense. 


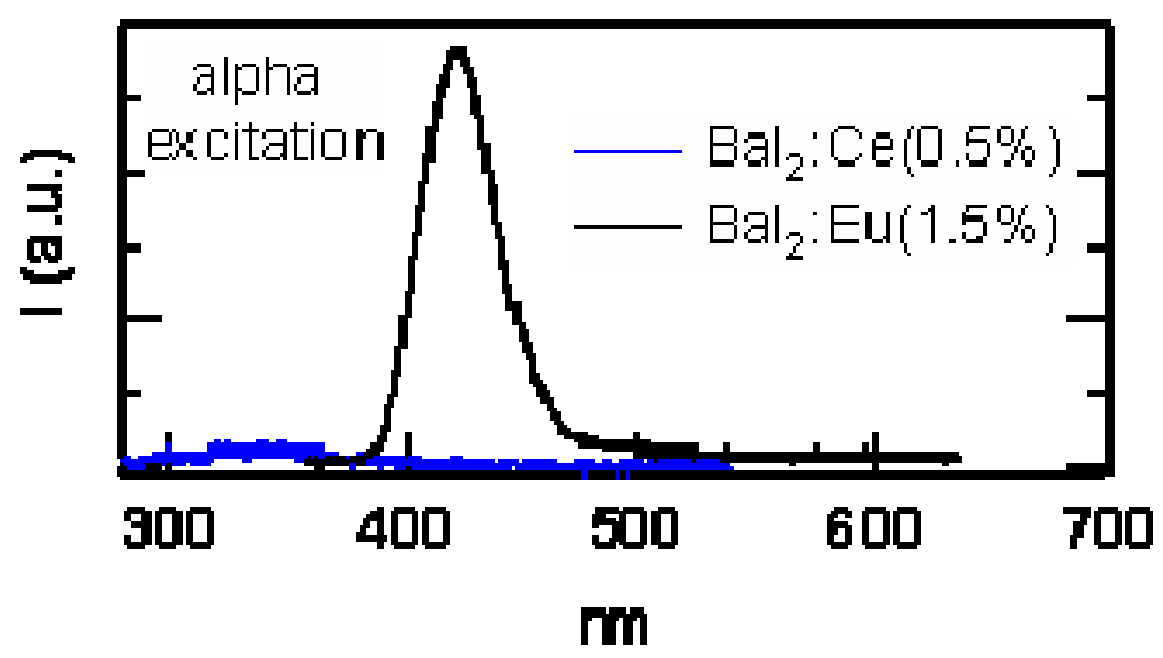

Fig. 4. Alpha-excited radioluminescence spectra acquired of a Ce- and a Eu-doped Barium Iodide crystal, both grown from zone refined materials. Light yields may be compared in an absolute sense.
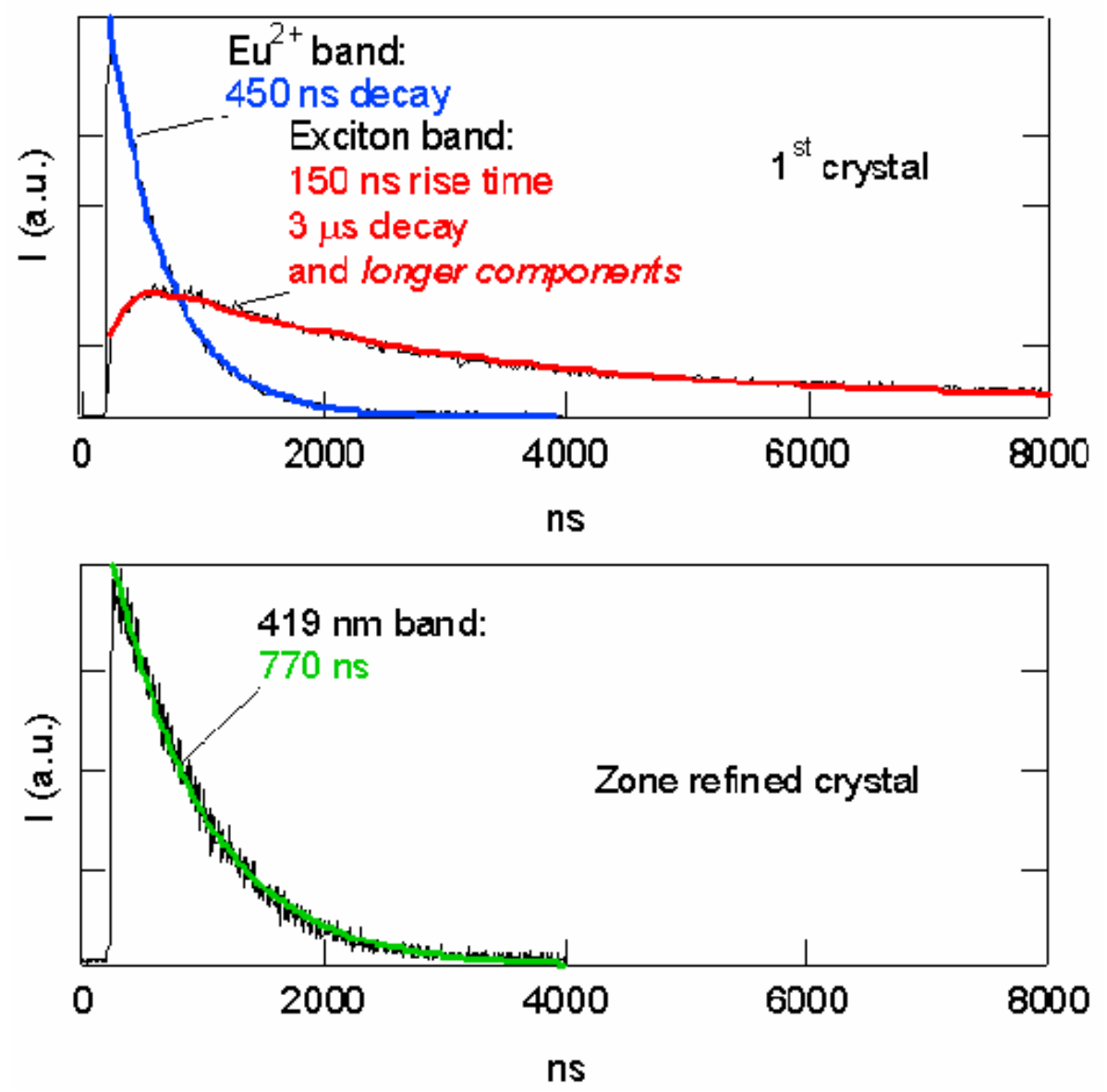

Fig. 5. Time-resolved luminescence decays acquired by excitation with $30 \mathrm{~ns}$ laser pulses at $266 \mathrm{~nm}$. 


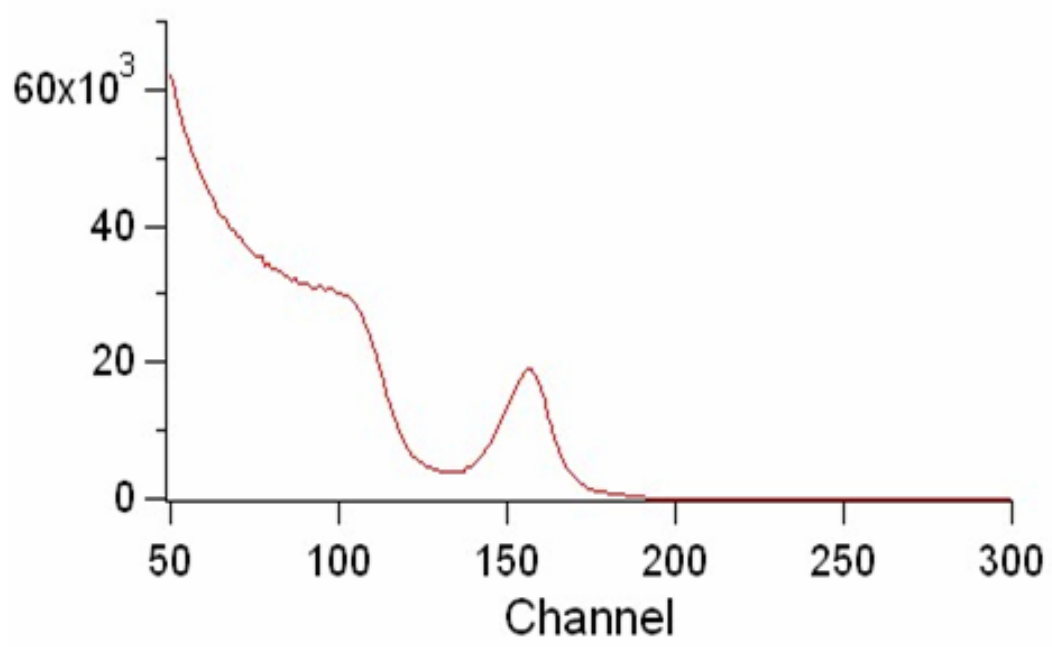

Fig. 6. Pulse height spectrum acquired of a $\mathrm{BaI}_{2}(\mathrm{Eu})(0.5 \mathrm{~mol} \%)$ crystal grown from as-received powders, yielding energy resolution at $662 \mathrm{keV}$ of $8 \%$.

Table 1. Beta-excited light yields are acquired with silicon detector (CCD camera) with flat spectral response and integrate the measured luminescence spectrum over 30s, while the pulse height spectra acquired at $662 \mathrm{keV}$ use a standard bialkali PMT, and a shaping time of 6-12 $\mu$ s.

\begin{tabular}{|l|c|c|}
\hline & $\begin{array}{c}\text { Light Yield } \\
\text { (all } \lambda \text { 's, no timing gate, } \\
\text { beta excitation) }\end{array}$ & $\begin{array}{c}\text { Energy Resolution } \\
(662 \mathrm{keV})\end{array}$ \\
\hline CsI(Tl) & 65,000 & $6.2 \%$ \\
\hline BGO & 9,750 & $12.7 \%$ \\
\hline $\mathrm{BaI}_{2}$ undoped (ZR) & 5,850 & -- \\
\hline $\mathrm{BaI}_{2}(\mathrm{Ce}) 0.5 \%$ (ZR) & 17,500 & $19 \%$ \\
\hline $\mathrm{BaI}_{2}$ (Eu) $0.5 \%$ (ZR) & 35,100 & $12 \%$ \\
\hline $\mathrm{BaI}_{2}(\mathrm{Eu}) 1.5 \%$ (ZR) & 39,000 & $13.5 \%$ \\
\hline $\mathrm{BaI}_{2}$ (Eu) $0.5 \%$ (not ZR) & 59,000 & $8 \%$ \\
\hline
\end{tabular}




\section{ACKNOWLEDGEMENTS}

This work was supported by the Domestic Nuclear Detection Office of the Department of Homeland Security and performed under the auspices of the U.S. Department of Energy by University of California, Lawrence Livermore National Laboratory under Contract W-7405-Eng-48.

\section{REFERENCES}

1. E.V.D. van Loef, P. Dorenbos, C.W.E. van Eijk, K. Kramer and H.H. Güdel, "High-energy-resolution scintillator: Ce ${ }^{3+}$ activated $\mathrm{LaBr}_{3}$, , Appl. Phys. Lett. 791573 (2001).

2. K.S. Shah, J. Glodo, M. Klugerman, W.M. Higgins, T. Gupta, P. Wong, “High energy resolution scintillation spectrometers,” IEEE Trans. Nucl. Sci 51, 2395-2999 (2004).

3. C. Fiorini, A. Gola, M. Zanchi, A. Longoni, P. Lechner, H. Soltau, L. Strüder, "Gamma-ray Spectroscopy with LaBr 3 :Ce Scintillator: Readout by a Silicon Drift Detector,” 2005 IEEE Nucl. Sci. Symp. (2005).

4. K. S. Shah, J. Glodo, M. Klugerman, W. Higgins, T. Gupta, P. Wong, W. W. Moses, S. E. Derenzo, M. J. Weber and P. Dorenbos, "LuI $: \mathrm{Ce}^{3+}$ - A new scintillator for gamma ray spectroscopy," IEEE Trans Nucl. Sci., 51, 2302 (2004).

5. M. D. Birowosuto, P. Dorenbos, C. W. E. van Eijk, K. W. Kr"amer and H.U. G"udel, "High-light-output scintillator for photodiode readout: LuI3:Ce ${ }^{3+}$,” J. Appl. Phys., 99, 123520 (2006).

6. R. Hofstadter, E. W. O’Dell, and S. T. Schmidt, IEEE Trans. Nucl. Sci. NS-11, 12 (1964).

7. J. Selling, M. D. Birowosuto, P. Dorenbos, S. Schweizer, “Europium-doped barium halide scintillators for x-ray and $\gamma$-ray detections," J. Appl. Phys., 101, 034901 (2007).

8. S. Kubota, J. Ruan (Gen), M. Itoh, S. Hashimoto, S., Sakuragi, , “A new type ofluminescence mechanism in large band-gapinsulators: proposal for fast scintillator materials,” Nucl. Inst.Meth. Phys. Res. A 289, 253-260 (1990).

9. P.A. Rodnyi, "Progress in fast scintillators," Radiation Measurements 33 605-614 (2001) 